\title{
An Ex Vivo Study of the Biological Properties of Porcine Aortic Valves in Response to Circumferential Cyclic Stretch
}

\author{
Kartik Balachandran, ${ }^{1}$ Suchitra Konduri, ${ }^{2}$ Philippe Sucosky, ${ }^{1}$ Hanjoong Jo, ${ }^{1,3}$ \\ and Ajit P. Yoganathan ${ }^{1}$ \\ ${ }^{1}$ The Wallace H. Coulter Department of Biomedical Engineering, Georgia Institute of Technology, Atlanta, GA 30332, USA; \\ ${ }^{2}$ School of Chemical and Biomolecular Engineering, Georgia Institute of Technology, Atlanta, GA 30332, USA; and \\ ${ }^{3}$ Department of Cardiology, Emory University, Atlanta, GA 30332, USA
}

(Received 8 January 2006; accepted 24 July 2006; published online: 10 October 2006)

\begin{abstract}
Normal physiological mechanical forces cause constant tissue renewal in aortic valve leaflets (AVL) while altered mechanical forces incite changes in their structural and biological properties. The current study aims at characterizing the remodeling properties of AVL subjected to cyclic circumferential stretch in a sterile ex vivo bioreactor. The leaflets cultured were stretched at a maximum rate of $300 \% \mathrm{~s}^{-1}$ corresponding to a $15 \%$ strain for $48 \mathrm{~h}$. Collagen, sulfated glycosaminoglycan (sGAG), and elastin contents of the stretched, fresh, and statically incubated leaflets were measured. Cusp morphology and cell phenotype were also examined. AVLs exposed to cyclic stretch showed a significant increase in collagen content $(p<0.05)$ when compared to fresh and statically incubated AVLs. sGAG content was significantly reduced in the stretched AVLs $(p<0.05)$ when compared to the fresh leaflets and was comparable between stretched and statically incubated AVLs. There was no statistically significant change in elastin content in all the three groups of AVLs $(p>0.05)$. Native aortic valve morphology was well preserved in stretched leaflets. Immunohistochemistry and immunoblotting studies showed an increased expression of $\alpha$-smooth muscle actin ( $\alpha$-SMA) in stretched leaflets while $\alpha$-SMA expression was reduced in statically incubated AVLs when compared to the fresh leaflets. To conclude, circumferential cyclic stretch altered the extracellular matrix remodeling activity of valvular cells, and consequently the extracellular matrix composition of the AVLs. Most interestingly, the contractile and fibrotic phenotypic expression of valve interstitial cells was enhanced. These results show that circumferential cyclic stretch is a possible mediator for AVL remodeling activity.
\end{abstract}

Keywords-Aortic valve leaflets, Contractile phenotype, Circumferential stretch, Uniaxial stretch bioreactor.

Address correspondence to Ajit P. Yoganathan, The Wallace H. Coulter Department of Biomedical Engineering, Georgia Institute of Technology, 313 Ferst Drive, Suite 1121, Atlanta, GA 30332-0535, USA. Electronic mail: ajit.yoganathan@bme.gatech.edu

\section{INTRODUCTION}

Cardiac valves play a crucial role in controlling blood flow through the body. ${ }^{6}$ Of particular interest in this study is the aortic valve, which at its most basic level controls blood flow between the left ventricle and the systemic circulation. In the United States, aortic valve disease has emerged as the third most common cardiovascular disease, ${ }^{17}$ with the number of aortic valve replacement surgeries steadily increasing over the past 20 years. ${ }^{33}$

The aortic valve is subjected to $30-40$ million cycles of opening and closing a year, which translates to approximately 3 billion cardiac cycles in a lifetime. ${ }^{26}$ During each cardiac cycle, the normal functional aortic valve interacts closely with the surrounding hemodynamic environment and is exposed to a myriad of mechanical forces such as transvalvular pressure, axial, shear and bending stresses, and cyclic flexure. ${ }^{26}$ Normal hemodynamic forces have been shown to cause constant tissue renewal in aortic valves, ${ }^{19}$ while altered mechanical forces are believed to induce changes in aortic valve biology possibly leading to valvular disease. ${ }^{16,30}$ However, the cellular and molecular events involved in these processes are not well characterized. Studies indicate that abnormal hemodynamics, especially hypertension, experienced by the valve leaflets cause tissue inflammation, which can lead to calcification, stenosis and ultimately valve failure ${ }^{1,15,23}$ Knowledge of the adaptive mechanisms of the aortic valves to altered hemodynamics will therefore improve our understanding on mechanotransduction of valvular disease progression and valve failure.

Mechanical forces modulate cell physiology, and have been shown to affect the biosynthetic activity of cells in tissue matrices. Previous studies on aortic valve mechanobiology have shown that porcine aortic valves cultured under normal hemodynamic conditions in a 
sterile ex vivo flow loop undergo constant renewal to maintain their native phenotypes. ${ }^{13}$ Porcine aortic valve endothelial cells exposed to $20 \mathrm{dyn} \mathrm{cm}^{-2}$ of steady laminar shear stress showed an organization of focal adhesion complexes and a cell alignment different than in static endothelial cell culture. ${ }^{4}$ Aortic valve leaflets exposed to hypertensive cyclic pressure showed increased collagen and sulfated glycosaminoglycan (sGAG) synthesis which varied in a magnitude and frequency-dependent manner. ${ }^{31}$ Studies exposing native porcine aortic valve leaflets to the isolated effects of flow, laminar steady shear stress, and constant static and cyclic pressure resulted in a reduced level of expression of $\alpha$-smooth muscle actin ( $\alpha$-SMA) ${ }^{29,31,32}$ This last finding might indicate the involvement of other forces in maintaining and regulating the contractile phenotype of aortic valve interstitial cells. To date the effect of cyclic stretch on aortic valves has not been studied.

The mechanical mode of cyclic stretching is important for normal aortic valve functionality as it allows, among other things, the valve leaflets to extend and form a coaptive seal with each other. However, it was seen that the valve leaflet tissue becomes less extensible and loses its ability to stretch rapidly with increasing age. ${ }^{15}$ This is mainly because collagen fibrillogenesis proceeds to maturity by increasing the diameter of some of the constituent collagen fibrils that require greater force to produce the same leaflet extension. ${ }^{8}$ Although the valve leaflet undergoes biaxial stretching in vivo, studies that investigate the effects of separate uniaxial (circumferential and radial) as well as biaxial cyclic stretch on the aortic leaflets would provide useful information on valve functionality or failure.

We hypothesize that the regulation of extracellular matrix remodeling as well as the expression of the contractile phenotype of aortic valve cells depend on the loading forces due to cyclic stretch. Here, we examined this hypothesis with a focus on the effect of physiological uniaxial circumferential cyclic stretch on the biosynthetic response of aortic valve leaflets using porcine aortic valve leaflets and an ex vivo tensile stretch bioreactor. Results from the current study will improve our understanding of the biological responses of native valves to circumferential stretching and will provide a good basis of comparison for conducting studies on radial cyclic stretching.

\section{MATERIAL AND METHODS}

\section{Tissue Harvest}

Fresh porcine aortic valves were obtained from a local abattoir (Holifield Farms, Covington, GA) following on-site dissection of the hearts within $30 \mathrm{~min}$ of slaughter. The valves were then transported to the laboratory in sterile, ice-cold Dulbecco's Phosphate Buffered Saline (DPBS; Sigma, St. Louis, MO).

Upon arrival to the laboratory, the three leaflets were excised from each valve and a rectangular section of tissue was isolated from the center of each leaflet. This tissue section was further divided into three rectangular sections having an aspect ratio of $12 \times 5 \mathrm{~mm}$ and oriented in the circumferential direction from the basal region. These three sections from each leaflet were randomly selected as fresh control, static control, and stretched leaflets, respectively (Fig. 1). Leaflet sections from the fresh control group were analyzed within $4 \mathrm{~h}$ following valve dissection. Leaflet sections from the static control group were statically incubated in Dulbecco's Modified Eagle Medium (DMEM; Sigma) at $37^{\circ} \mathrm{C}$ for the duration of the experiment $(48 \mathrm{~h})$. Leaflet sections from the stretched group had loops sutured to the $5 \mathrm{~mm}$ ends and were suspended in DMEM in a tensile stretch bioreactor. ${ }^{10}$ These samples were then cyclically stretched at a frequency of $1.167 \mathrm{~Hz}$ (equivalent to 70 beats per minute) to $15 \%$ strain at a maximum strain rate of $300 \% \mathrm{~s}^{-1}$ at $37^{\circ} \mathrm{C}$. The temporal strain waveform imposed (Fig. 2a) in the circumferential direction was derived from the variations of the leaflet length in the circumferential direction over one cardiac cycle. ${ }^{25,26}$ The extension of the leaflet corresponded to diastole $(2 / 3$ of the cardiac cycle), while the unstretched state of the leaflet corresponded to systole ( $1 / 3$ of the cardiac cycle). The gradients of the extension and relaxation approximated those experienced in vivo. ${ }^{25}$ The above waveform resulted in a tissue extension of $1.8 \mathrm{~mm}$ at a maximum rate of extension of $36 \mathrm{~mm} \mathrm{~s}^{-1}$. Each experiment was run for $48 \mathrm{~h}$, with 12-15 leaflet sections for each experimental run.

\section{Stretch Bioreactor System and Validation}

The stretch bioreactor used in this study (Fig. 2b) was developed by Engelmayr et al. ${ }^{10}$ The device

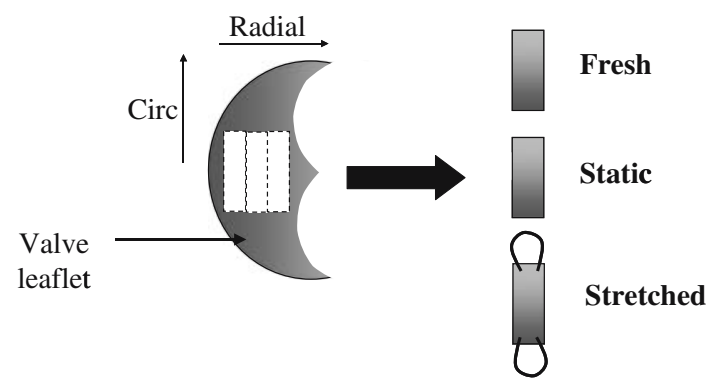

FIGURE 1. Preparation of aortic valve leaflets for the experiment. Three sections were cut out in the circumferential direction from the base, belly and tip regions of the aortic valve leaflet. These sections were randomly chosen as stretched, fresh and static leaflet groups. Fresh sections were processed immediately. Static control sections were incubated for $48 \mathrm{~h}$ in DMEM. Stretched sections were stretched for $48 \mathrm{~h}$. 

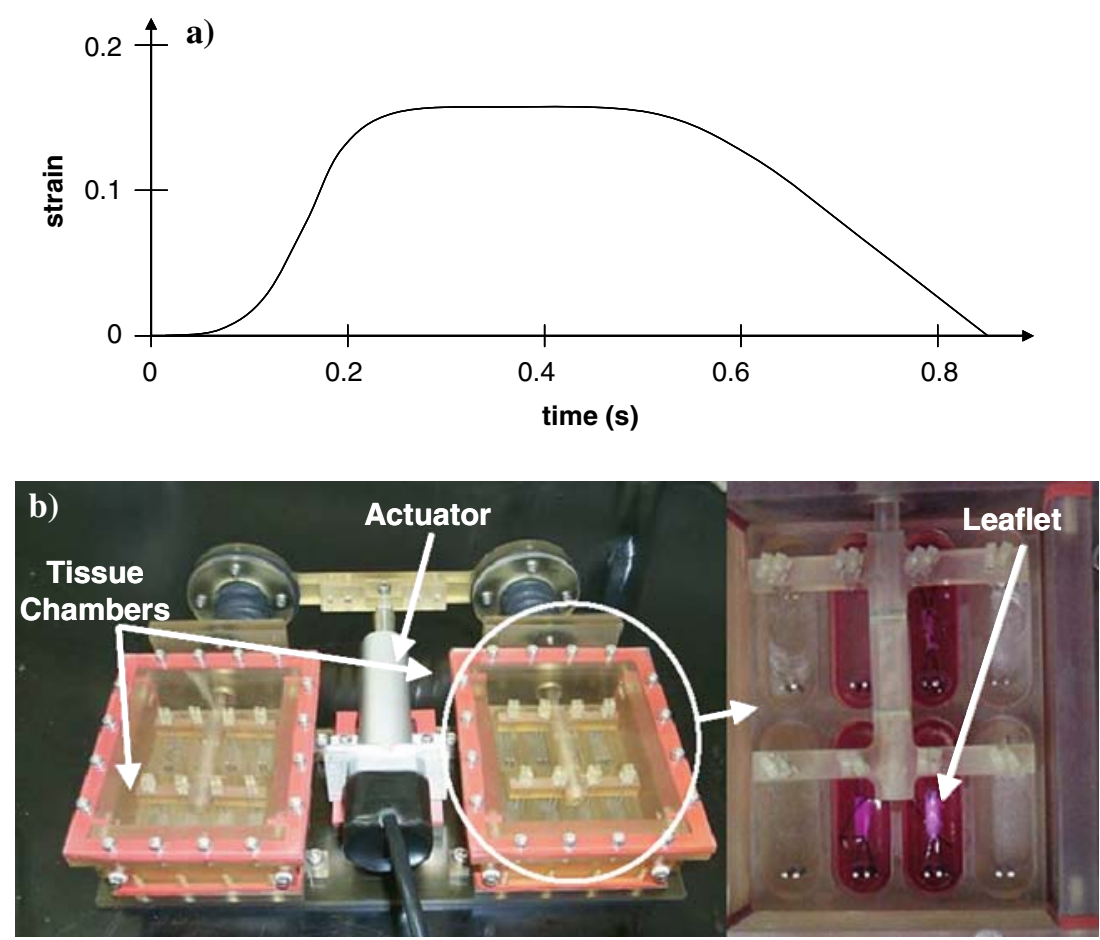

FIGURE 2. (a) Loading curve used in this study. A near physiologic loading curve ${ }^{25}$ was input to the actuator to stretch the leaflets. (b) Ex vivo tensile stretch bioreactor used in this study. A magnified image of the tissue chamber is shown on the right showing eight tissue wells, with aortic valve leaflets in four of the wells.

consists of two tissue chambers coupled via a crossarm to a centrally positioned linear actuator (Ultra Motion LLC, Mattituck, NY). Each chamber consists of eight culture wells within which two "stationary posts" are positioned. The linear motion of the actuator is translated into uniaxial motion of two "moving posts" via the cross-arm which bifurcates to two moving arms. These moving arms then enter each individual bioreactor chamber and terminate at each culture well with the two "moving posts" mentioned above. The interior of the chamber is protected from external contamination by a neoprene-lined lid. The bioreactor was constructed from polysulfone, which is thermally and chemically stable, while the lids were constructed using acrylic. The device was assembled using 18-8 stainless steel screws (McMaster Carr, Cleveland, $\mathrm{OH}$ ) and fabricated by Apollo Precision Inc. (Plymouth, MN). The motor input waveform (Fig. 2a) was downloaded to the actuator controller card using the Si-Programmer software (Ultra Motion LLC).

After assembly, the bioreactor was validated for uniformity of strains on the leaflet sections in all the culture wells. This was achieved by placing dye markers (Shandon tissue marking dye, Thermo Electron Corp, Pittsburgh, PA) on aortic valve leaflets and capturing the distance between the markers under uniaxial cyclic stretch using a high-speed camera capture technique developed in our laboratory. ${ }^{12}$ The bioreactor was validated at input strains of 15 and $25 \%$.

In order to determine if the stretch bioreactor induced a shear stress on the leaflet surface due to the relative motion of the leaflet with respect to the surrounding media, a computational fluid dynamics (CFD) model was designed, validated and used to predict the leaflet wall shear stress under the imposed loading curve in the stretch bioreactor.

For the experiment, the bioreactor was sterilized using hydrogen peroxide in a gas-plasma sterilizer (Advanced Sterilization Products, Irvine, CA), and assembled aseptically in a sterile laminar flow hood (Fisher Hamilton, Two Rivers, WI). The culture wells were filled with $8 \mathrm{ml}$ of serum-free DMEM supplemented with $50 \mathrm{mg} \mathrm{l}^{-1}$ ascorbic acid, $3.7 \mathrm{~g} \mathrm{l}^{-1}$ sodium bicarbonate, $1 \%(\mathrm{v} / \mathrm{v})$ nonessential amino acid solution, and $1 \%(\mathrm{v} / \mathrm{v})$ antimycotic-antibiotic solution (all reagents from Sigma). Each tissue section was suspended in the medium by the two sutured loops - one end was looped over the stationary posts while the other end was looped over the moving post. The bioreactor was then placed in an incubator (Fisher Scientific, Hampton, $\mathrm{NH}$ ) maintained at $37^{\circ} \mathrm{C}, 5 \% \mathrm{CO}_{2}$ atmosphere at normal atmospheric pressure and the leaflets were stretched for $48 \mathrm{~h}$. 


\section{Tissue Processing}

At the end of the experiment, each leaflet section was further cut into three equal rectangular sections. These resulting samples were dehydrated for $48 \mathrm{~h}$ at room temperature in a vacuum oven (Yamato Scientific America, San Francisco, CA) prior to digestion in pepsin (Sigma), protease (Sigma) and proteinase K (Sigma), for analyzing collagen, sulfated glycosaminoglycan (sGAG), and elastin contents, respectively. All digestions were carried out in a water bath maintained at $37^{\circ} \mathrm{C}$. The total amount of collagen, sGAG and elastin was determined using the Sircol $^{\mathrm{TM}}$ Collagen assay kit (Biocolor, UK), Bly$\operatorname{scan}^{\mathrm{TM}}$ sGAG assay kit (Biocolor), and Fastin ${ }^{\mathrm{TM}}$ Elastin assay kit (Biocolor), respectively. These kits used quantitative dye-binding methods to determine the total quantity of the respective ECM component in the sample.

Valve leaflet samples from additional experiments were isolated for analysis of tissue morphology and cell phenotype. For histological and immunohistochemical analyses, leaflet sections were fixed in $10 \%$ neutral buffered formalin (Fisher Scientific, Suwanee, GA) for $24 \mathrm{~h}$, saturated in $70 \%$ ethanol, processed in a tissue processor (Shandon Pathcenter enclosed Tissue Processor) in descending grades of ethanol, embedded in paraffin and cut into $5 \mu \mathrm{m}$ sections.

\section{Morphological Characterization}

Routine Hematoxylin and Eosin ( $\mathrm{H} \&$ E) stain was used to examine gross tissue structure and indicate any changes in the structural compositions of the leaflets. $\mathrm{H} \& \mathrm{E}$ staining was done on an autostainer (Leica, Vashaw Scientific, Norcross, GA). Picrosirius red staining was performed to examine collagen fiber structure and morphology. The protocol was as follows. Deparaffinized slides were incubated in Weigert's hematoxylin (Sigma) followed by picrosirius red solution for one hour. The slides were then washed in acidified water, three changes of $100 \%$ ethanol, and xylene before being coverslipped. These slides were viewed at $100 \times$ using a Nikon imaging microscope (Nikon Inc., Melville, NY). A Q-imaging camera, Retiga 1300C (I-Cube, Glen Burnie, MD) and its bundled software Q-Capture Pro was used to acquire images of the tissue sections. $\mathrm{H} \& \mathrm{E}$ images were recorded using normal white light while picrosirius red images were recorded with circularly polarized light.

\section{Characterization of Cell Phenotype}

$\alpha$-smooth muscle cell actin ( $\alpha$-SMA) immunohistochemistry (IHC) was used to detect myofibroblasts and smooth muscle cells. The staining provided information on changes in cell phenotype between the three experimental treatments. The protocol was as follows. Deparaffinized slides were blocked using $1 \%$ gelatin/ phosphate buffered saline (PBS; Sigma) for $30 \mathrm{~min}$. The slides were then incubated in mouse monoclonal anti $\alpha$-SMA primary antibody (Sigma) in $1 \%$ Bovine Serum Albumin (BSA)/PBS (Sigma) for one hour. The sections were saturated in biotinylated horse anti-mouse $\operatorname{IgG}$ (Vector laboratories) in 1\% BSA/PBS, and 2\% normal horse serum (Vector laboratories) for $30 \mathrm{~min}$. Avidin-D Texas red (Vector Laboratories) fluorochrome was applied to the sections after the secondary antibody was washed off. The sections were then counter stained with $0.25 \mu \mathrm{g} \mathrm{ml}^{-1} 4^{\prime}$, 6-Diamidino-2-phenylindole (DAPI; Sigma), coverslipped and stored at $4^{\circ} \mathrm{C}$. Slides were subsequently imaged at $200 \times$ using a TR-FITC-DAPI triple filter under a mercury lamp.

\section{Image Analysis}

$\mathrm{H} \& \mathrm{E}$ images were analyzed by measuring the fibrosa: spongiosa length ratio, and the fibrosa: ventricularis length ratio. $\alpha$-SMA immunopositive cells appear red with cell nuclei counterstained blue, while the color of collagen fibers changes from green to yellow to orange to red as the fiber thickness (and consequently maturity) increases. The proportion of $\alpha$-SMA positive cells were therefore assessed by using the Image $\mathbf{J}$ program (NIH, Bethesda, MD) to determine the relative area coverage of $\alpha$-SMA positive red staining compared to the area coverage of entire leaflet section. The relative proportion of the different maturity of the collagen fibers in the leaflet section were calculated using published methods ${ }^{2}$. Basically, picrosirius red images were first imported into Matlab 7.01 (The Mathworks, Natick, MA) and converted into their hue, saturation and value components. Only the hue components were retained, and a histogram of hue frequency was obtained from the 8-bit hue images that contained 256 possible colors. The colors were defined as follows: red 2-9 and 230-256; orange 10-38; yellow 39-51; and green 52-128. All other hue values corresponded to interstitial space and were confirmed by visual inspection. The number of pixels within each hue range were determined and expressed as a percentage of the total number of collagen pixels and plotted.

\section{Immunoblotting Analysis}

The rest of the leaflet sections were processed for analysis by immunoblotting. Leaflets were homogenized and sonicated in ice-cold lysis buffer (in mmol $1^{-1}$ : Tris-HCl 20 (pH 7.5), EGTA 5, $\mathrm{NaCl} 150$, glycerol-phosphate $20, \mathrm{NaF} 10$, sodium orthovanadate 
1, $1 \%$ Triton $\mathrm{X}-100,0.1 \%$ Tween-20 and protease inhibitors) to extract proteins from the tissue, and then centrifuged at 14,000 rpm to pellet cellular debris. The supernatant was assayed for protein concentration using the DC Protein Assay (Bio-Rad, Hercules, CA) and equal aliquots of tissue lysates were resolved on a $10 \%$ SDS-PAGE gel and subsequently blotted to a polyvinyldifluoride (PVDF) membrane (Millipore, Billerica, MA). Membranes were incubated with a mouse anti $\alpha$-SMA primary antibody (Sigma) at $4{ }^{\circ} \mathrm{C}$ overnight $(1: 1000)$, and then with a goat anti-mouse alkaline phosphatase-conjugated secondary antibody (Bio-Rad). CDP-Star reagent (NE Biolabs, Ipswich, MA) was added and chemiluminescence of the resulting product was detected using photographic paper. GAPDH antibody (Santa Cruz Biotech, Santa Cruz, CA) was used as a loading control (1:5000). Pixel densities of $\alpha$-SMA immunoreactive bands were then analyzed using the Image $\mathbf{J}$ program and normalized by the intensities of the GAPDH immunoreactive bands.

\section{Analysis of Tissue Culture Medium}

As a follow-up to determine if sGAGs were leaching out from the leaflet in the DMEM, six samples of the DMEM were analyzed before and after the experiment. The Blyscan ${ }^{\mathrm{TM}}$ sGAG assay kit (Biocolor) used in the ECM analysis was used to detect sGAGs in the medium.

\section{Statistical Analysis}

The data for collagen, sGAG and elastin contents were expressed as mean plus one standard error of the mean. A total of 27 leaflets were analyzed for the ECM assays, 8 leaflets for the histological and immunohistochemical studies, and 10 leaflets for the Western blotting studies. The quantitative data from the ECM assays, image analysis and immunoblotting were analyzed first using ANOVA followed by pairwise comparisons with the Tukey post-hoc test. A $p$-value of less than 0.05 was used as a measure of statistical significance. When only two groups were being compared, data were analyzed using student $t$-tests with a $p$-value of less than 0.05 indicating statistical significance. All statistical analyses were performed using Minitab (Minitab R14, Minitab Inc. State College, PA).

\section{RESULTS}

\section{Validation of Stretch Bioreactor}

The stretch bioreactor produced a uniform output strain across the leaflet of $14.80 \pm 0.53 \%$ for $15 \%$ input strain, and $25.48 \pm 0.53 \%$ for $25 \%$ input strain. Performing a student t-test between input and output signals revealed no significant difference $(p>0.05$, $n=16$ ), indicating that the bioreactor could accurately and uniformly reproduce the specified input strain across the entire leaflet section.

The peak steady-state wall shear stress experienced by the leaflet as calculated from CFD was $2.5 \mathrm{dyn} \mathrm{cm}^{-2}$. This is small compared to the peak $79 \mathrm{dyn} \mathrm{cm}^{-2}$ experienced by the leaflet under physiological flow conditions during systole as measured in our laboratory, ${ }^{28}$ and it can be assumed that stretch is the dominant force acting on the leaflet.

\section{Extracellular Matrix Content ${ }^{1}$}

A significant increase in collagen content was observed in stretched leaflets when compared to fresh and static control leaflets $(p<0.05, n=27)$. Stretched leaflets showed a $90 \%$ and $161.5 \%$ increase in collagen content in comparison to fresh and static controls, respectively. There was no significant difference in collagen content between fresh and static control leaflets $(p>0.05, n=27)$. sGAG content was found to be decreased by $40 \%$ in stretched leaflets when compared to fresh valve leaflets $(p<0.05, n=27)$, while sGAG content was comparable between stretched and static control leaflets $(p>0.05, n=27)$. ANOVA showed that there was no significant difference ( $p>0.05, n=27$ ) between treatment groups for elastin content. The results for the ECM assays are graphically depicted in Fig. 3.

Analysis of the conditioned medium at the end of experimental duration revealed a statistically significant increase $(p<0.05, n=6)$ in sGAG secreted in to the media $\left(5.83 \pm 0.56 \mu \mathrm{g} \mathrm{ml}^{-1}\right.$ of DMEM) compared to the fresh media $(1.08 \pm 0.30)$.

\section{Cell Morphology and Phenotype}

H \& E staining (Fig. 4a) of the leaflets showed preserved three-layered leaflet architecture in stretched leaflets consistent with fresh and static control leaflets. The cell nuclei were found to be intact with no tears in the tissue section. Image analysis showed no significant difference $(p>0.05, n=8)$ in the fibrosa-spongiosa length ratio as well as the fibrosa-ventricularis length ratio (Fig. 5a) indicating that there was no gross damage to leaflets structure.

Picrosirius red staining (Fig. 4b) of the leaflets showed that collagen architecture was preserved in

\footnotetext{
${ }^{1}$ ECM refers only to pepsin-soluble collagen, sulfated glycosaminoglycans and elastin
} 


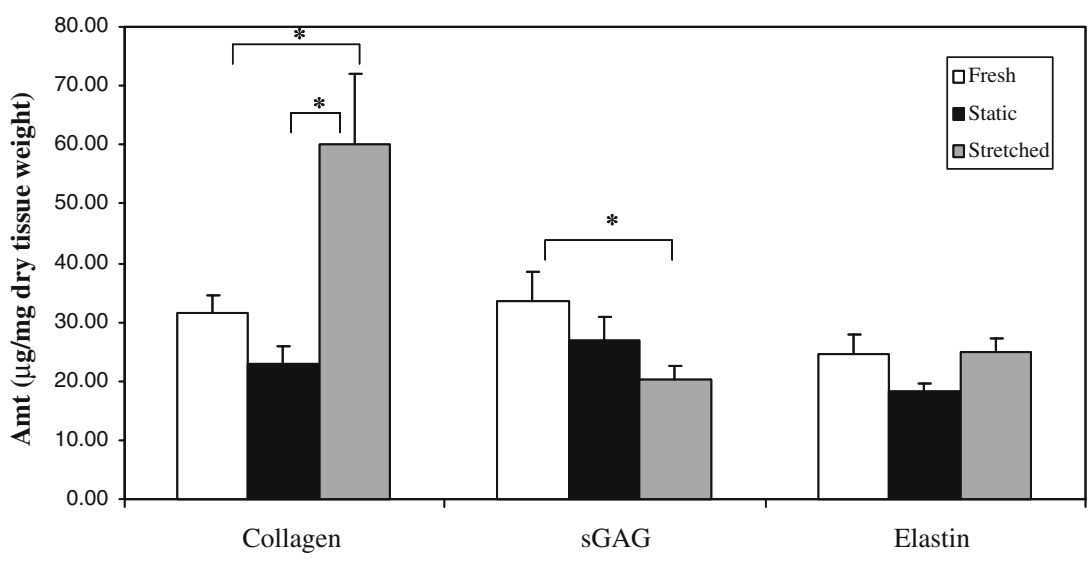

FIGURE 3. Cyclic stretch increased collagen, decreased sGAG, while not affecting elastin contents in aortic valve leaflets. The leaflets were exposed to physiologic circumferential cyclic stretch (15\% at heart rate of $70 \mathrm{bpm})$ or static control conditions for $48 \mathrm{~h}$. The amounts of collagen, sGAG and elastin in the leaflets exposed to static or stretching were compared to those of fresh leaflets. The data were normalized by tissue dry weight and expressed as a mean value plus one standard error of the mean ( $\left.{ }^{*} p<0.05, n=27\right)$.
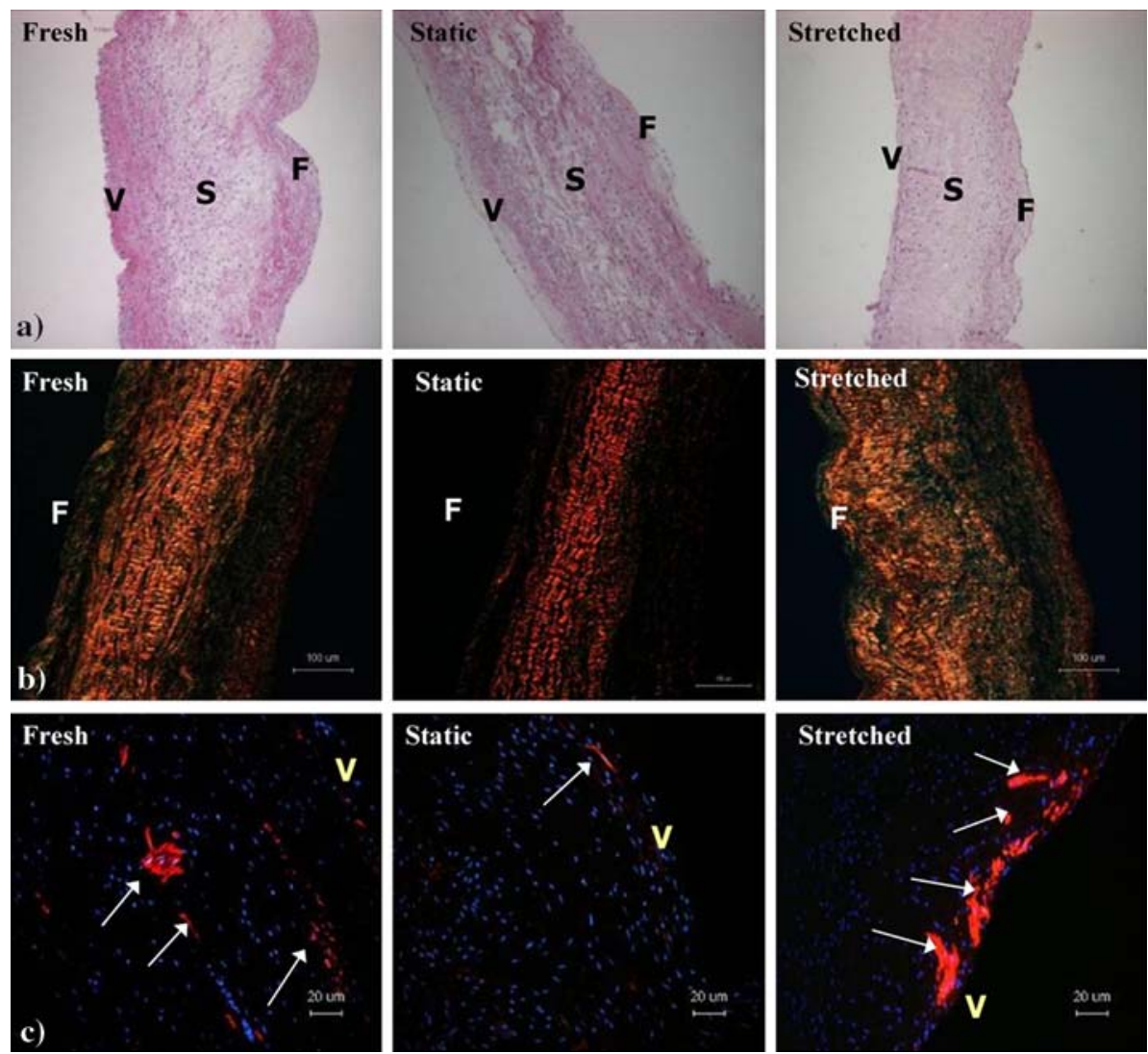

FIGURE 4. (a) Cyclic stretch did not damage tissue structure and morphology. H \& E stained images of fresh, static and stretched aortic valve leaflets are depicted here. Cytoplasm was stained pink and cell nuclei were stained blue. The representative images show the three-layered morphology of the leaflets was intact in all three groups (F - fibrosa, S - spongiosa, V - ventricularis). (b) Cyclic stretch maintained native collagen architecture. Picrosirius red images of fresh, static and stretched aortic valve leaflets are shown. Mature collagen fibers were stained orange-red. Layered collagen morphology of leaflets was observed and crimp was preserved in stretched leaflets (F - fibrosa). (c) Cyclic stretch increased $\alpha$-smooth muscle actin ( $\alpha$-SMA)-positive cells in the ventricularis side of the aortic valve leaflets. Fresh, static and stretched leaflets were examined by $\alpha$-SMA IHC. Actin was stained red and cell nuclei were counterstained blue. Increased expression of $\alpha$-SMA was observed in stretched leaflets, and $\alpha$-SMA was reduced in static leaflets ( $\mathrm{V}$ - ventricularis). 
fresh, static as well as stretched leaflets. The layered structure of collagen along with the crimp in the collagen fibers could clearly be seen in the stretched leaflets, demonstrating that the stretching motion did not damage valvular collagen. These findings demonstrated that uniaxial cyclic stretch of the leaflets did not damage leaflet morphology and maintained native ECM structure and cellular composition.

Additionally, hue analysis of the picrosirius red images (Fig. 5b) revealed that the proportion of newly synthesized (green) fibers in statically incubated leaflet sections was significantly decreased by $36.5 \%$ $(p<0.05, n=8)$ compared to fresh leaflets. There was no significant difference in green fibers $(p>0.05$, $n=8$ ) between fresh and stretched leaflets, however, mature (red) collagen fibers significantly increased by $101.4 \%$ in stretched leaflets compared to fresh leaflets.

The immunohistochemical study with $\alpha$-SMA (Fig. 4c), a marker of myofibroblast-like or smooth muscle cell-like valvular interstitial cells ${ }^{2}$, revealed that cyclic stretch up-regulated the expression of $\alpha$-SMA, while static incubation down-regulated the expression of $\alpha$-SMA. This was corroborated by image analysis (Fig. 5c) of the images, which showed significantly increased $(p<0.05, n=8) \alpha$-SMA immunopositive staining in stretched leaflets compared to fresh leaflets,
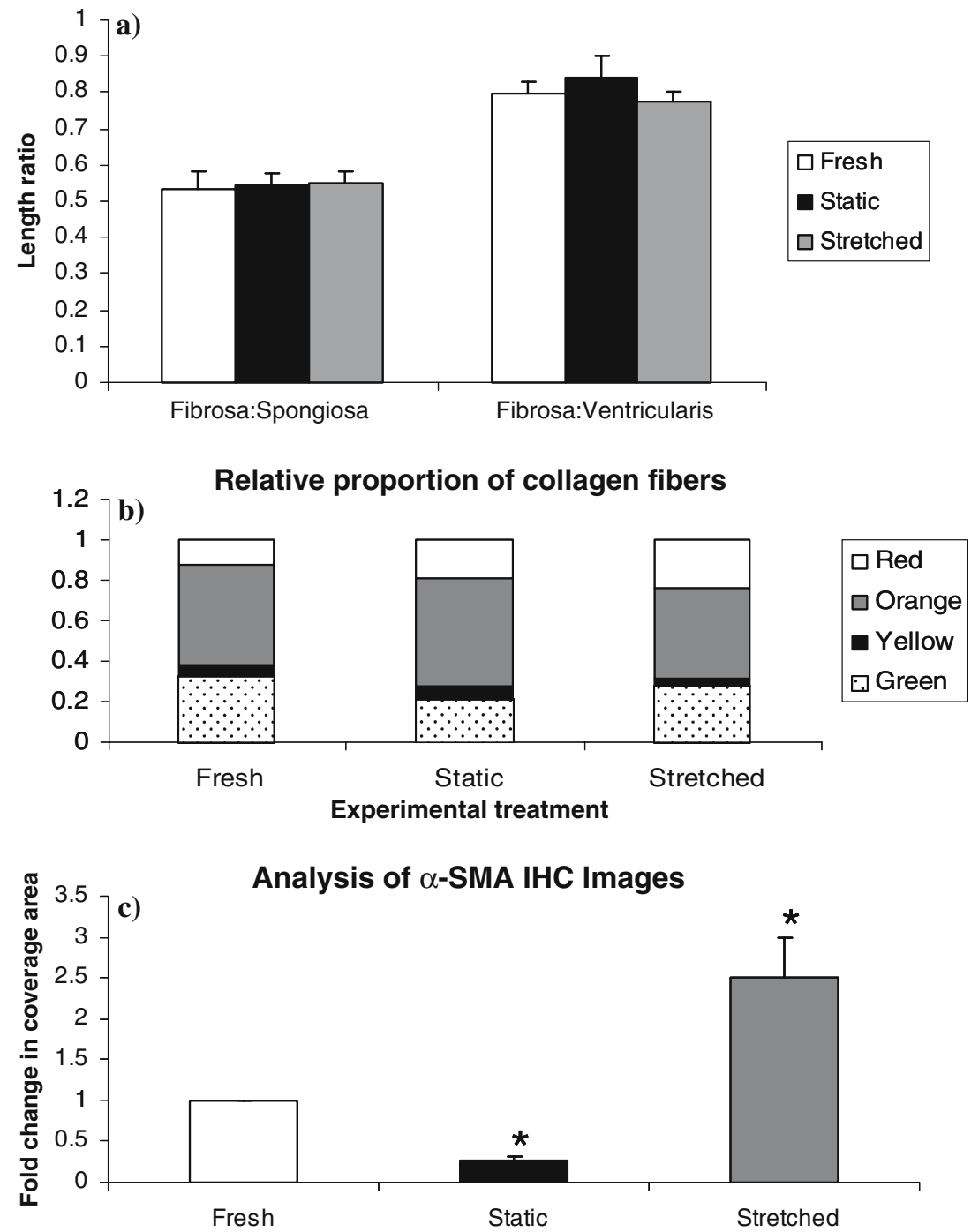

FIGURE 5. (a) Fibrosa-to-spongiosa ratio and fibrosa-to-ventricularis ratio remained unchanged. There was no significant difference $(p>0.05, n=8)$ in the relative thicknesses of the fibrosa, spongiosa and ventricularis layers between fresh, static and stretched leaflets. (b) Static leaflets exhibited reduced levels of newly synthesized collagen fibers. Newly synthesized fiber proportion was reduced in static leaflets signifying reduced levels of collagen synthesis. Newly synthesized fiber proportion was comparable between fresh and stretched leaflets, but the proportion of mature fibers was greater in stretched leaflets. (c) Cyclic stretch increases $\alpha$-smooth muscle actin in the aortic valve leaflets. $\alpha$-SMA immunopositive staining coverage was increased in stretched leaflets and reduced in static leaflets when compared with fresh leaflets. 
and significantly reduced $(p<0.05, n=8) \alpha$-SMA staining in static leaflets compared to fresh leaflets. Another interesting observation was that the $\alpha$-SMA positive cells in the stretched leaflets were predominantly found in the ventricularis region of the aortic valve leaflet while there was little or no $\alpha$-SMA expression in the fibrosa. In contrast, $\alpha$-SMA positive cells were randomly distributed in all the three regions of the fresh and static leaflets.

The above finding was further reinforced by the Western blotting results (Fig. 6). There was a $54.5 \%$ increase $(p<0.05, n=10)$ in $\alpha$-SMA content of stretched leaflets and $34.9 \%$ decrease $(p<0.05$, $n=10)$ in statically incubated leaflets when compared to fresh leaflets. These results together suggest that cyclic stretching induces phenotypic change of valve interstitial cells toward myofibroblast-like cells in a side-dependent manner.

\section{DISCUSSION}

\section{Effect of Cyclic Stretch on ECM Components}

The current study investigated the effects of isolated circumferential cyclic stretch on the biological responses of aortic valve leaflets using a sterile ex vivo stretch bioreactor. Initial validation of the bioreactor showed that strains were uniform across the leaflet section. It was also validated that there was negligible wall shear stress on the leaflet, and therefore cyclic stretch was the only force acting on the leaflet.
It was revealed that the collagen content of the aortic valve leaflets stretched to $15 \%$ was increased when compared to fresh and static control leaflets, while sGAG content was decreased in stretched leaflets compared to fresh leaflets. Furthermore, elastin content was comparable between the stretched and fresh leaflets. This suggests that exposure to isolated effects of circumferential cyclic stretch at physiological levels has altered the extracellular matrix composition, consequently, altering the valve remodeling.

Collagen fibers, densely populated in parallel alignment along the circumferential direction of the leaflet, form the load bearing structure of the leaflet during each cardiac cycle. ${ }^{18,26}$ The increase in collagen suggests that the leaflets adapt to altered mechanical loading due to the effects of isolated cyclic stretch, by either increasing synthesis, or decreasing degradation of collagen. The image analysis of the picrosirius red staining (Fig. 5b) showed comparable proportions of newly synthesized fibers, and hence collagen synthesis in both fresh and stretched leaflets. The increased proportion of mature (red) fibers in stretched leaflets therefore leads us to believe that it is the reduced degradation of collagen that causes the overall total increase in collagen content. The preserved crimp in collagen fibers in the stretched leaflet, as evidenced by picrosirius red staining also implies that the native structure of collagen was not damaged by the stretching motion.

This data is in agreement with a study conducted by $\mathrm{Ku}$ et al. ${ }^{14}$ where porcine aortic valve interstitial cells were cultured on a collagen substrate and stretched $48 \mathrm{~h}$. For cells subjected to $14 \%$ cyclic stretch, collagen
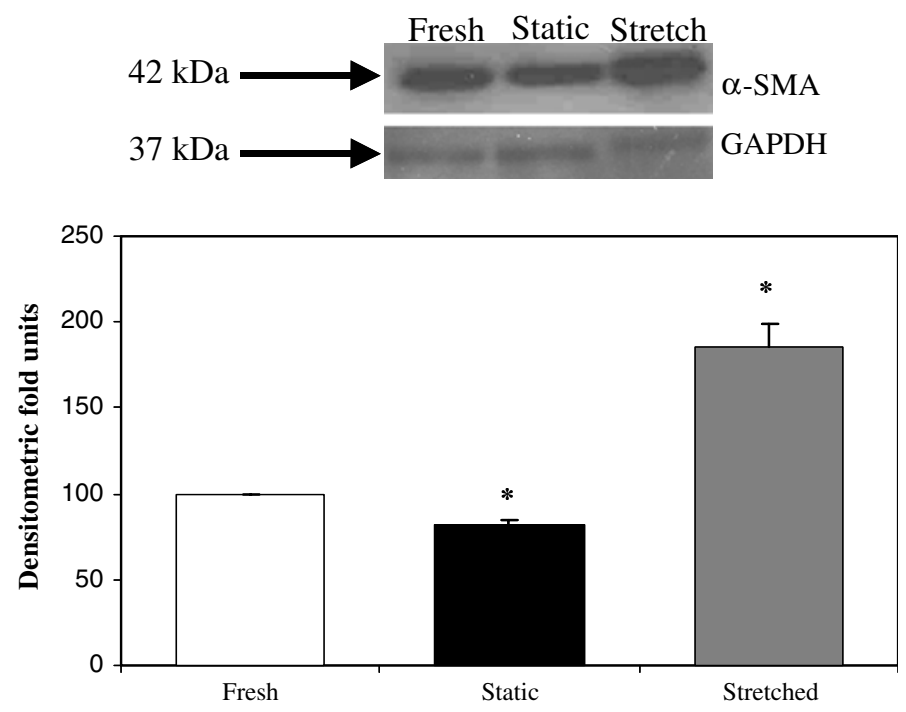

FIGURE 6. Cyclic stretch increased $\alpha$-smooth muscle actin in the aortic valve leaflets. Fresh, static and stretched aortic valve leaflets were analyzed by Western blot with $\alpha$-SMA antibody and analyzed by densitometry. When compared to fresh leaflets, $\alpha$-SMA expression decreased in static leaflets, but increased in stretched leaflets $\left({ }^{*} p<0.05, n=10\right)$. Anti-GAPDH antibody was used as a loading control. 
incorporation in the cell layer was approximately $170 \%$ of unstretched cells. No significant changes were observed at time points less than $48 \mathrm{~h}$. This provides further evidence that $48 \mathrm{~h}$ of culture is a necessary and sufficient time point to observe changes in the valvular ECM.

The semi-fluidic nature of the sGAG in the spongiosa layer of the leaflet gives the leaflet considerable plasticity. ${ }^{26}$ It is therefore believed that the purpose of sGAG might be (i) to help reduce leaflet stresses by lubricating the relative motion of the other two layers as the leaflet deforms, and (ii) to dampen compressive forces during diastole. ${ }^{7}$ The absence of compressive stresses in this study, with the stretch being carried out at atmospheric pressure, might therefore explain the reduced levels of sGAG observed in the stretched leaflet compared to fresh leaflets. Additionally, analysis of the DMEM at the end of experimental duration showed a statistically significant increase in sGAG content in the media. This suggests that cyclic stretching of the aortic valve leaflets resulted in secreting or leaching of the sGAG from the leaflets into the media, as the various layers of the leaflet rub against each other. A study conducted by Grande-Allen et al. ${ }^{11}$ also showed a loss of GAG from explanted porcine bioprosthetic valves due to leaching even under normal storage conditions. This suggests that there is a high propensity for sGAGs to be forced out of the native aortic valve either during static incubation or leaflet motion. In contrast, aortic valve leaflets when exposed to normal physiological forces undergo constant sGAG renewal by the interstitial cells. $^{13}$

In the ventricularis layer of the leaflet, the major extracellular component is elastin in the form of fibers that are oriented primarily along the radial direction. Their primary role of elastin fibers is to maintain a circumferential collagen fiber orientation and recoil the collagen fibers to their initial state after the external load has been released. ${ }^{27}$ Since the valve leaflets were cyclically stretched along the circumferential direction at physiological rates in the current study, it is possible that the elastin fibers were not "activated" beyond their normal levels and consequently additional synthesis of elastin was not required, explaining why elastin content remained unchanged between fresh and stretched leaflets.

The above findings reinforce the fact that valve cells require mechanical stimulation to maintain a balance between synthesis and degradation of ECM components. This occurred without undue damage to the normal tri-layered structure of the aortic valve leaflet as the relative thicknesses of the fibrosa, spongiosa, and ventricularis were maintained (Fig. 5a).

\section{Effect on Cell Phenotype}

Valvular interstitial cells are mainly comprised of fibroblasts, myofibroblasts, and smooth muscle cells that exhibit different phenotypes depending on the microenvironment surrounding the cells. Expression of $\alpha$-SMA was associated with the contractile phenotype of smooth muscle cells ${ }^{24}$ and myofibroblast-like cells, ${ }^{2}$ and hence was chosen as a marker for examining the interstitial cell phenotype in the present study. Studies have shown that the expression of $\alpha$-SMA was decreased by changes in culture conditions and mechanical and biochemical stimulation in cultured vascular cells. ${ }^{21,22}$ Studies in our laboratory have shown that $\alpha$-SMA expression decreased in response to isolated effects of pressure and shear forces. ${ }^{13,29,31}$ Similar behavior was observed in valvular interstitial cells in $3 \mathrm{D}$ static gel culture. ${ }^{5}$

The current study revealed via IHC (Fig. 5c) and Western blotting (Fig. 6) that circumferential cyclic stretch increases the expression of $\alpha$-SMA in aortic valve leaflets. It is therefore speculated that cyclic stretch is a regulator of the contractile and fibrotic phenotypes of aortic valve interstitial cells. A reversible equilibrium between F-actin depolymerization and G-actin polymerization exists in the cells of aortic valves. The contractile motion of the leaflets due to stretch may have caused a shift in this equilibrium towards polymerization of $\mathrm{G}$-actin to form $\mathrm{F}$-actin fibrils, which is detected as $\alpha$-SMA immunopositive cells.

These findings also lead to the possibility that in the normal hemodynamic environment, the up-regulating effect of stretch on $\alpha$-SMA expression may be counterbalanced by the down-regulating effects on $\alpha$-SMA expression of pressure and shear stress. This provides an additional possible explanation for the fact that aortic valves cultured in normal hemodynamic forces maintain a constant $\alpha$-SMA expression. ${ }^{13}$

Another interesting observation was that $\alpha$-SMA immunopositive cells occurred preferentially in the ventricularis of the stretched leaflet. This is in contrast to fresh and static leaflets which exhibited an apparent random distribution of $\alpha$-SMA through all the three layers of the valve leaflet. This may provide insights into specific regions or "hot spots" on the aortic valve leaflet where there is a focal response to mechanical forces. In a study by Simmons et al. ${ }^{20}$ spatial heterogeneity of aortic valve endothelial phenotypes was speculated to contribute to the focal susceptibility for calcific aortic valve lesion development, implicating the endothelium as a regulator of valvular calcification. The current study shows the potential focal and sidespecific response of the interstitial cells of the aortic valve to mechanical forces. In addition, $\alpha$-SMA is a 
known marker for mesenchymal cells, ${ }^{3,9}$ and the expression of $\alpha$-SMA could also indicate regions of endothelial cell to mesenchymal cell transdifferentiation. It is clear that further side-specific study is needed in this area as it may provide insights into how valves sense and transmit mechanical forces.

\section{CONCLUSION}

The current study demonstrated that circumferential cyclic stretch altered the extracellular matrix remodeling activity of native porcine aortic valve leaflets. These results combined with results from previous ex vivo studies show that the changes in aortic valve leaflet biology depend intimately on the influence of local hemodynamic environmental factors. Further research is needed to understand the mechanistic pathways behind these biological changes. The knowledge gleaned from such studies will ultimately allow for elucidation of mechanisms of aortic valve pathogenesis leading to valve failure.

\section{ACKNOWLEDGMENTS}

This study was supported by the National Science Foundation through the ERC program at Georgia Institute of Technology under award number EEC9731643. The authors would like to acknowledge Dr. Michael S. Sacks and George C. Engelmayr Jr. for the design of the stretch bioreactor. The authors also thank Mr. Holifield for donating the porcine hearts for this research, Tracey Couse for her technical assistance, and Jason Xenakis, Christina Clements and Christina Duden for providing assistance in the study.

\section{REFERENCES}

${ }^{1}$ Agmon, Y. et al. Aortic valve sclerosis and aortic atherosclerosis: different manifestations of the same disease? Insights from a population-based study. J. Am. Coll. Cardiol. 38(3):827-834, 2001.

${ }^{2}$ Aikawa, E. et al. Human semilunar cardiac valve remodeling by activated cells from fetus to adult: implications for postnatal adaptation, pathology, and tissue engineering. Circulation 113(10):1344-1352, 2006.

${ }^{3}$ Armstrong, E. J. and J. Bischoff. Heart valve development: endothelial cell signaling and differentiation. Circ. Res. 95(5):459-470, 2004.

${ }^{4}$ Butcher, J. T. et al. Unique morphology and focal adhesion development of valvular endothelial cells in static and fluid flow environments. Arterioscler. Thromb. Vasc. Biol. 24(8):1429-1434, 2004.

${ }^{5}$ Butcher, J. T. and R. M. Nerem. Porcine aortic valve interstitial cells in three-dimensional culture: comparison of phenotype with aortic smooth muscle cells. J. Heart. Valve Dis. 13(3):478-485, 2004: (discussion 485-486).
${ }^{6}$ Butler, D. L., S. A. Goldstein and F. Guilak. Functional tissue engineering: the role of biomechanics. J. Biomech. Eng. 122(6):570-575, 2000.

${ }^{7}$ Christie, G. W. Anatomy of aortic heart valve leaflets: the influence of glutaraldehyde fixation on function. Eur. J. Cardiothorac. Surg. 6(Suppl 1):S25-32, 1992: discussion S33.

${ }^{8}$ Christie, G. W. and B. G. Barratt-Boyes. Age-dependent changes in the radial stretch of human aortic valve leaflets determined by biaxial testing. Ann Thorac Surg. 60(2 Suppl):S156-8, 1995: discussion S159.

${ }^{9}$ DeRuiter, M. C. et al. Embryonic endothelial cells transdifferentiate into mesenchymal cells expressing smooth muscle actins in vivo and in vitro. Circ. Res. 80(4):444-451, 1997.

${ }^{10}$ Engelmayr, G. C. Jr. et al. A novel bioreactor for the dynamic flexural stimulation of tissue engineered heart valve biomaterials. Biomaterials 24(14):2523-2532, 2003.

${ }^{11}$ Grande-Allen, K. J. et al. Loss of chondroitin 6-sulfate and hyaluronan from failed porcine bioprosthetic valves. $J$. Biomed. Mater. Res. A. 65(2):251-259, 2003.

${ }^{12} \mathrm{He}$, Z. et al. In vitro dynamic strain behavior of the mitral valve posterior leaflet. J Biomech Eng. 127(3):504-511, 2005.

${ }^{13}$ Konduri, S. et al. Normal physiological conditions maintain the biological characteristics of porcine aortic heart valves: an ex vivo organ culture study. Ann. Biomed. Eng. 33(9):1158-1166, 2005.

${ }^{14} \mathrm{Ku}, \mathrm{C}$. H. et al. Collagen synthesis by mesenchymal stem cells and aortic valve interstitial cells in response to mechanical stretch. Cardiovasc. Res. 71(3):548-556, 2006.

${ }^{15}$ Mohler, E. R. III et al. Bone formation and inflammation in cardiac valves. Circulation. 103(11):1522-1528, 2001.

${ }^{16}$ Quick, D. W. et al. Collagen synthesis is upregulated in mitral valves subjected to altered stress. Asaio. J. 43(3):181186, 1997.

${ }^{17}$ Rajamannan, N. M., B. Gersh and R. O. Bonow. Calcific aortic stenosis: from bench to the bedside-emerging clinical and cellular concepts. Heart 89(7):801-805, 2003.

${ }^{18}$ Sacks, M. S., D. B. Smith and E. D. Hiester. The aortic valve microstructure: effects of transvalvular pressure. $J$. Biomed. Mater. Res. 41(1):131-141, 1998.

${ }^{19}$ Schneider, P. J. and J. D. Deck. Tissue and cell renewal in the natural aortic valve of rats: an autoradiographic study. Cardiovasc. Res. 15(4):181-189, 1981.

${ }^{20}$ Simmons, C. A. et al. Spatialheterogeneity of endothelial phenotypes correlates with side-specific vulnerability to calcification in normal porcine aortic valves. Circ. Res. 96(7):792-799, 2005.

${ }^{21}$ Stegemann, J. P. and R. M. Nerem. Phenotype modulation in vascular tissue engineering using biochemical and mechanical stimulation. Ann. Biomed. Eng. 31(4):391-402, 2003.

${ }^{22}$ Stegemann, J. P. and R. M. Nerem. Altered response of vascular smooth muscle cells to exogenous biochemical stimulation in two- and three-dimensional culture. Exp. Cell. Res. 283(2):146-155, 2003.

${ }^{23}$ Stewart, B. F. et al. Clinical factors associated with calcific aortic valve disease. Cardiovascular Health Study. J. Am. Coll. Cardiol. 29(3):630-634, 1997.

${ }^{24}$ Taylor, P. M. et al. The cardiac valve interstitial cell. Int. J. Biochem. Cell Biol. 35(2):113-118, 2003.

${ }^{25}$ Thubrikar, M. et al. The elastic modulus of canine aortic valve leaflets in vivo and in vitro. Circ Res. 47(5):792-800, 1980. 
${ }^{26}$ Thubrikar, M. The Aortic Valve. CRC Press, Boca Raton, Fla 221Thubrikar M., (1990) The Aortic Valve. CRC Press, Boca Raton, Fla., pp 221.

${ }^{27}$ Vesely, I. The role of elastin in aortic valve mechanics. $J$. Biomech. 31(2):115-123, 1998.

${ }^{28}$ Weston, M. W., D. V. LaBorde and A. P. Yoganathan. Estimation of the shear stress on the surface of an aortic valve leaflet. Ann. Biomed. Eng. 27(4):572-579, 1999.

${ }^{29}$ Weston, M. W. and A. P. Yoganathan. Biosynthetic activity in heart valve leaflets in response to in vitro flow environments. Ann. Biomed. Eng. 29(9):752-763, 2001.

${ }^{30}$ Willems, I. E. et al. Structural alterations in heart valves during left ventricular pressure overload in the rat. Lab. Invest. 71(1):127-133, 1994.
${ }^{31}$ Xing, Y. et al. Cyclic pressure affects the biological properties of porcine aortic valve leaflets in a magnitude and frequency dependent manner. Ann. Biomed. Eng. 32(11):1461-70, 2004.

${ }^{32}$ Xing, Y. et al. Effects of constant static pressure on the biological properties of porcine aortic valve leaflets. Ann. Biomed. Eng. 32(4):555-562, 2004.

${ }^{33}$ Yacoub, M. H. and L. H. Cohn. Novel approaches to cardiac valve repair: from structure to function: Part II. Circulation 109(9):1064-1072, 2004. 